\title{
PORCINE ANAESTHESIA FOR ADVANCED TRAUMA OPERATIVE MANAGEMENT (ATOM)
}

\author{
H. BADDOO, F. AHIAKU, E. FORDJUOR, I. WULFF, D. AKUOKU and D. KWAMI
}

\author{
Department of Anaesthesia, University of Ghana Medical School, Accra, Ghana
}

Author for correspondence: Dr Henry Baddoo

E-mail: hbaddoo@yahoo.com

Conflict of interest: None declared

\begin{abstract}
SUMMARY
Anaesthesia has been provided for pigs for the Advanced Trauma Operative Management Course which was first held at the Korle $\mathrm{Bu}$ Teaching Hospital in February 2005. As of January 2006, 16 animals have been anaesthetized. Acepromazine $(1 \mathrm{mg} / \mathrm{kg})$ and atropine $(0.05 \mathrm{mg} / \mathrm{kg})$ were used for premedication. IM ketamine $(30 \mathrm{mg} / \mathrm{kg}$ ) and isoflurane (in oxygen) were used for the anaesthesia and the animals were intubated and ventilated with positive pressure ventilation. No neuromuscular blocking agents were used. Morphine (average 3 doses of $10 \mathrm{mg}$ ) was used for analgesia. Systolic blood pressure ranged between $80 \mathrm{mmHg}$ and $115 \mathrm{mmHg}$ and diastolic pressure ranged between $45 \mathrm{mmHg}$ and $80 \mathrm{mmHg}$. Large amounts of IV fluids were given during the procedure. At the end of surgery, the animals were euthanized by increasing the concentration of isoflurane and administering potassium chloride. Out of the 14 cases reported on, 13 animals survived to the end of all the surgical procedures. 1 animal had a cardiac arrest during the last procedure (inflicting a laceration to the heart) and could not be resuscitated.
\end{abstract}

\section{KEY WORDS}

Porcine anaesthesia, acepromazine, atropine, ketamine, isoflurane, invasive monitoring

\section{INTRODUCTION}

The Advanced Trauma Operative Management Course was first held at the Korle $\mathrm{Bu}$ Teaching Hospital in February 2005. Since then two further courses have been held - the second in August, 2005 and the third in November 2005. In this course, injuries were inflicted on the pigs and the surgeons taking part in the course were taught how to deal with these injuries.

Anaesthesia is required for these animals before the injuries are inflicted. The animals have to be kept anaesthetized and kept stable throughout the procedures. The technique of anaesthesia used is basically the same as that used for the ATOM course in Hartford, Connecticut, USA. No neuromuscular blocking agents are used, but the animals are anaesthetized with isoflurane, made deep enough to be intubated and then ventilated with intermittent positive pressure ventilation.

The purpose of this paper is to describe the techniques of anaesthesia used, the difficulties encountered and the outcome of anaesthesia, which is hoped would be of use to any group that needs to administer porcine anaesthesia.

\section{PREPARATION OF THE ANIMALS}

The protocol for the ATOM Animal Laboratory was used for anaesthetizing the pigs. Mr. Bill Dyckman, Research Technologist of the ATOM course from Hartford, USA was of invaluable help in teaching us how to anaesthetize the pigs during the first course.

\section{Technique of Anaesthesia}

The sizes of the pigs used are approximately $50 \mathrm{~kg}$. The animals were fasted overnight. The animals were first given a dose of acepromazine $1 \mathrm{mg} / \mathrm{kg}$ and atropine $0.05 \mathrm{mg} / \mathrm{kg}$. These were given intramuscularly in the muscle mass just behind the ears. About 15minutes later, intramuscular ketamine $30 \mathrm{mg} / \mathrm{kg}$ was given in two divided doses separated by an interval of $10 \mathrm{~min}$ utes. The animals became anaesthetized soon after the ketamine was given and on this regime maintained a good airway. The animals were then put on a trolley, shaved and then transferred onto an operating table. A pulse oximeter probe was attached to the ears and anaesthesia was deepened by giving, via a specially designed face mask, up to $3 \%$ Isoflurane in oxygen. The muscle tones of the limbs were assessed from time to time and when the animals became quite floppy, it was deemed that the animals were deep enough to be intubated. While the animals were being deepened on isoflurane, a cannula was inserted into a vein on the ears; this was connected to an infusion of Ringers lactate.

\section{Intubation}

To intubate the animals the jaws were first retracted using two metal rings, one on the upper jaw and one on 
the lower jaw. The tongue can also be retracted using sponge holding forceps.

The laryngoscopes were then inserted. The laryngoscopes' blades used were a \#11 Miller blade which is a long straight blade. Once the epiglottises were seen the blades were inserted behind the epiglottis and the larynx were visualized. Pigs easily go into laryngeal spasm, and to prevent this, the cords were sprayed with $10 \%$ lidocaine spray. To do this a $5 \mathrm{ml}$ syringe containing lidocaine was used, which was attached to the dilator from a Swan-Ganz sheath set. This dilator provides a firm long device that made it possible to direct the lidocaine from a distance, very close to the cords. About $3 \mathrm{ml}$ of lidoocaine were used. Once the cords were sprayed with lidocaine, the trachea was intubated using an $8 \mathrm{~mm}, 7.5 \mathrm{~mm}$ or $7.0 \mathrm{~mm}$ cuffed endotracheal tube. Intubation tended tricky, and special manoeuvres were applied to make it easier. The tubes were initially inserted with the concave part of the tube upwards (as one would do in a human) but as the tubes were advanced, one rotates it through approximately 180 degrees so that the convex part of the tube faces upwards.

Once the animals were intubated, anaesthesia was maintained on $1.5-2 \%$ isoflurane in oxygen and the animals were ventilated on a tidal volume of $12 \mathrm{ml} / \mathrm{kg}$ and at a rate of approximately 12 breaths per minute. Intramuscular morphine $10 \mathrm{mg}$ was given for analgesia and this was repeated if an animal showed any signs of pain while under anaesthesia.

\section{Intravenous fluids}

A cut down was done to cannulate the right or left external jugular vein and the right or left carotid artery. An incision $(5-10 \mathrm{~cm})$ was made two finger breadths lateral to the trachea in the mid portion of the neck. The external jugular vein and the carotid arteries were isolated and cannulated. The carotid artery was connected to a pressure transducer system and the external jugular had an infusion of Ringers lactate going through it which could be infused at a high rate during the times when blood loss was severe.

A lot of fluids were needed, partly because of the blood loss involved and partly because 'third-space losses 'is significant in pigs. When hypotension occurred inspite of adequate fluid therapy, boluses of ephedrine $5 \mathrm{mg}$ or adrenaline (1 in 10,000), 25 micrograms were given.

\section{Resuscitation}

Two of the animals went into ventricular fibrillation while the chest was open and this was corrected by defibrillating the heart directly with a DC shock of 20 joules.

\section{Monitoring}

Oxygen saturation (pulse oximetry) and invasive blood pressure were monitored. It was planned in the future to also monitor temperature. ECG was not monitored as it would be difficult to apply conventional electrodes on to the skin of the pigs.

\section{End of Surgery}

At the end of surgery, the animals were euthanized by turning the isoflurane up to $3 \%$ and giving a lethal dose of $40 \mathrm{mmol}$ of potassium chloride.

\section{RESULTS}

Sixteen animals have been anaesthetized for the course; 8 during the first course which was held on two days in February 2005, 5 during the second course (one trial run and 4 for the course) in August 2005 and 3 during the third course in November 2005.

One of the anaesthesia records was missing, and one of the animals anesthetized was used for a trial run in which the cardiac injuries were not inflicted. The results of the remaining 14 cases will therefore be presented. Table 1 shows the weight, physiological parameters of the pigs and the duration of surgery.

Table 1: Weight, physiological parameters of the pigs and the duration of surgery

\begin{tabular}{|l|l|}
\hline PARAMETER & MEAN (RANGE) \\
\hline Weight $(\mathrm{kg})$ & $50.5(40.6-58.7)$ \\
SBP (mmHg) & $89(80-115)$ \\
DBP (mmHg) & $52(45-80)$ \\
Lowest HR (beats/minute) & 70 \\
Highest HR (beats/minute) & 165 \\
Duration of surgery (hours) & $3.46(2.66-4.66)$ \\
\hline
\end{tabular}

SBP - Initial systolic blood pressure after insertion of carotid arterial line; DBP - Initial diastolic blood pressure after insertion of carotid arterial line; HR - Heart rate of animals during surgery

Table 2: Interval between initial injection of ketamine and start of surgery

\begin{tabular}{|l|l|}
\hline DATE & $\begin{array}{l}\text { TIME IN HOURS. } \\
\text { (RANGE) }\end{array}$ \\
\hline DAY 1, FEB 2005 & $2.63(2.0-3.26)$ \\
DAY 2, FEB 2005 & $1.55(1.33-1.75)$ \\
AUGUST 2005 & $2.13(1.83-2.5)$ \\
NOV 2005 & $2.32(1.92-2.72)$ \\
\hline
\end{tabular}

The amount of IV fluids given during surgery ranged from 6 litres to 17 litres with a mean of 12.9 litres. 
Thirteen of the 14 animals survived till the end of all the procedures and were euthanized. One of these animals went into ventricular fibrillation on opening the chest but was successfully resuscitated by given a DC shock of 20 joules directly to the heart. One animal had a cardiac arrest during the last procedure - inflicting a laceration to the heart. Inspite of cardiac massage, defibrillation, adrenaline and other drugs, the animal could not be resuscitated.

Doses of morphine given during anaesthesia ranged from 2 doses of $10 \mathrm{mg}$ to 5 doses of $10 \mathrm{mg}$. The most common amount given was 3 doses of $10 \mathrm{mg}$. Ephedrine was given to support the blood pressure in 6 animals and adrenaline (epinephrine) was used in 5 animals.

\section{DISCUSSION}

Giving general anaesthesia to pigs has several potential problems. Excessive salivation, relative lack of peripheral veins except on the earlobe, laryngeal anatomy that makes endotracheal intubation difficult for the inexperienced and the tendency to go into laryngeal spasm are some of them. The animals are also quite difficult to restrain to give the initial intramuscular injections and on a few occasions the needle came out of the tissues before the full injection was given. Under anaesthesia, pigs are prone to develop problems such as hyperthermia, hypothermia, hypotension and hypoventilation. Pigs can also develop Malignant Hyperthermia.

Some literature recommends that the animals are fasted for 24 hours. With the ATOM course regime they are fasted overnight. This period of fasting was adequate and there were no instances of regurgitation or vomiting during anaesthesia in our cases.

Mean systolic blood pressure of $89 \mathrm{mmHg}$ and mean diastolic pressure of $52 \mathrm{mmHg}$ were obtained when the arterial lines were initially inserted. One has to take into account the fact that the animals would have been anaesthetized with isoflurane for a while before the arterial line is inserted and isoflurane causes vasodilatation which would tend to lower the blood pressure. As mentioned, the animals tend to need a lot of intravenous fluids and a mean of 12.9 litres of fluid was used for each animal. In the ATOM course protocol, a premixed bag of phenylephrine was used as an infusion to titrate to maintain the blood pressure when hypotension occurs inspite of adequate fluid replacement. As phenylephrine is not available in our institution boluses of ephedrine were used in its place. Ephedrine and phenylephrine are both sympathomimetic amines. There are however some differences in their pharmacological properties. Ephedrine has both $\alpha$ and $\beta$ effects and causes an increase in cardiac output and heart rate.
Peripheral vascular resistance is more or less unchanged. Phenylephrine on the other hand has strong $\alpha$ effects and only weak $\beta$ effects. There is little effect on cardiac output. Blood pressure is increased due to vasoconstriction causing an increase in peripheral vascular resistance, and heart rate is slowed due to stimulation of the baroreceptors. Adrenaline was also used on a number of occasions. Morphine was given for analgesia and it was found that 3 doses of $10 \mathrm{mg}$ was the amount usually needed. Morphine was chosen as the analgesic to use as it is cardiovascularly stable and it is cheap.

Temperature was not measured during anaesthesia but one suspected that hypothermia was prone to occur since the animals were anaesthetized for long periods in an air-conditioned room. Sometimes one was overcautious to make sure the animals were anaesthetized in good time with the result that the animals were under anaesthesia for quite some time before surgery began. This would add to the risk of hypothermia. From the analysis, it was seen that 1 hour 20 minutes after the administration of ketamine was adequate time to get the animal ready for surgery. We would therefore suggest that the timing of anaesthesia is planned so that ketamine is given one and a half hour before surgery is due to commence.

\section{Possible future management strategies}

For future courses efforts should be made to measure the temperature of the animals to see whether hypothermia is a significant problem, so, measures can be instituted to prevent hypothermia. At present the central venous pressure (CVP) of the animals is not measured. Measuring the CVP would help determine more accurately how much fluid to give the animals.

An article in 'Anaesthesiology' ${ }^{3}$ reported that vasopressin enhances survival in a liver trauma model with uncontrolled and otherwise lethal haemorrhagic shock in pigs. In future, vasopressin could be used instead of phenylephrine or ephedrine, to support the blood pressure in addition to fluids, especially after the inferior vena cava injury, to see whether it keeps the animals more stable before the cardiac injuries are performed.

\section{ACKNOWLEDGEMENTS}

We would like to acknowledge the tremendous support of the following persons: Prof. Lenworth Jacobs, Director of EMS/Trauma Programme, Founder of ATOM Course, Hartford Hospital, USA, Dr Stephen Luk, Medical Director, ATOM Course, Hartford Hospital, Mr William Dyckman, Research Technologist, Hartford Hospital, Mr Mark Heydenburg, Director, Medical Equipment Services, International Aid, Surgical Col- 
leagues, Korle $\mathrm{Bu}$ Teaching Hospital and Nursing Staff, ATOM course.

\section{REFERENCES}

1. Jacobs L.M., Ronald Gross, Stephen Luk. Advanced Trauma Operative Management Course Manual Publishers: Woodbury: Cine-med Inc 2004 Editor: Stephen A. Green

2. Veterinary Anaesthesia and Pain Management Secrets Publishers: Philadelphia: Hanley and Belfus Inc Medical Publishers 2002
3. Stadlbauer, Karl H., Horst G. Wagner, Claus Raedler, Wolfgang G Voelckel, Volker Wenzel, Annette C. Kirsmer, Guenter Klima, Klaus Rheinberger, Walter Nussbaumer, Daniel Pressmar and Karl Lindner Vasopressin but not fluid resuscitation enhances survival in a liver trauma model with uncontrolled and otherwise lethal hemorrhagic shock in pigs. Anaesthesiology 2003; 98 : 699-704 